\title{
PROBABILITY DENSITY BASED CLASSIFICATION AND RECONSTRUCTION OF ROOF STRUCTURES FROM 3D POINT CLOUDS
}

\author{
Y. Dehbi ${ }^{1 *}$ S. Koppers ${ }^{1}$, L. Plümer ${ }^{2}$ \\ ${ }^{1}$ Institute of Geodesy and Geoinformation, University of Bonn, Germany \\ dehbi@igg.uni-bonn.de, koppers@uni-bonn.de \\ ${ }^{2}$ Faculty of Geosciences and Environmental Engineering, Southwest Jiaotong University, Chengdu, China \\ pluemer@swjtu.edu.cn
}

KEY WORDS: city model, probability density function, roof, dormer, machine learning, support vector machine.

\begin{abstract}
:
3D building models including roofs are a key prerequisite in many fields of applications such as the estimation of solar suitability of rooftops. The accurate reconstruction of roofs with dormers is sometimes challenging. Without careful separation of the dormer points from the points on the roof surface, the estimation of the roof areas is distorted in a most characteristic way, which then let the dormer points appear as white noise. The characteristic distortion of the density distribution of the defects by dormers in comparison to the expected normal distribution is the starting point of our method. We propose a hierarchical method which improves roof reconstruction from LiDAR point clouds in a model-based manner separating dormer points from roof points using classification methods. The key idea is to exploit probability density functions (PDFs) to reveal roof properties and design skilful features for a supervised learning method using support vector machines (SVMs). Properties of the PDFs of measures such as residuals of model-based estimated roof models are used among others. A clustering step leads to a semantic segmentation of the point cloud enabling subsequent reconstruction. The approach is tested based on real data as well as simulated point clouds. The latter allow for experiments for various roof and dormer types with different parameters using an implemented simulation toolbox which generates virtual buildings and synthetic point clouds.
\end{abstract}

\section{INTRODUCTION}

3D city models in the level-of-detail 2 (LoD2) of the standardized exchange format CityGML (Gröger et al., 2012) have nowadays a wide range of applications comprising urban planing and traffic simulation among others. A state of the art review on the applications of 3D city models is given by Biljecki et al. (2015). The automatic generation of virtual models and in particular the reconstruction of the is-built-state of existing ones is still an intensive research topic. A survey of urban reconstruction algorithms is presented by Musialski et al. (2012) where different data, sensors and methods are shown. A further review on various reconstruction methods for urban modeling from laser scanning data has been recently published by Wang et al. (2018).

Due to the high relevance of roof models in many fields of applications, the 3D reconstruction of rooftops from aerial data, e.g. LiDAR or photogrammetric point clouds, is a well studied subject of research. In this context, top-down, bottom-up and even hybrid methods are employed. Most of these methods focus on roof models without taking their superstructures, i.e. dormers and chimneys, into consideration. Top-down methods are suffering from big structures which smear the estimated models wheras bottom-up methods have difficulties to identify small structures from the data. Roof structures such as dormers and chimneys represent outliers regarding to the according roof model. The occurrence of such outliers complicates the robust modeling of roofs. Hence, our key idea is to examine the impact of such structures on the roof determination in order to derive roof models with a high accuracy. From an urban planning point of view and with

\footnotetext{
${ }^{*}$ Corresponding author
}

a view to a more precise classification of the type of use, dormers are of great importance because they indicate the value of the building and the type and intensity of its use.
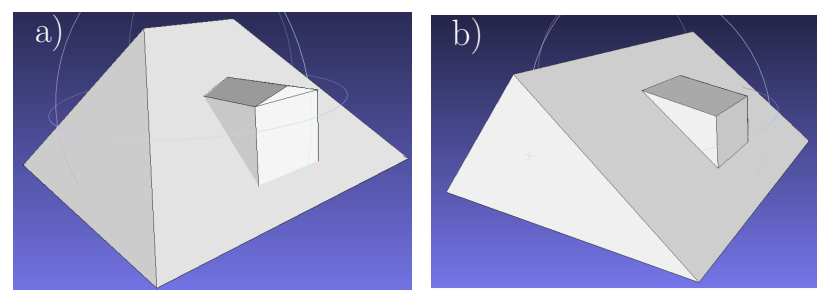

Figure 1. Roof models with different dormer types: a) gabled dormer and b) shed dormer.

The main contribution of this paper is a novel approach which classifies and reconstructs roofs and their structures based on designed statistical features derived from probability density functions (PDFs). The characteristic distortion of the density distribution of the defects caused by dormers in comparison to the expected normal distribution is the starting point of our method. The method is in particular able to detect and differentiate between gabled and shed dormers as depicted in Figure 1 a) and b) respectively. To this aim, we implemented a simulation environment which enables us to analyze roof dormer parameters in a systematic way. The implemented simulation toolbox allows for the generation of point clouds and gives the possibility to generate various roof structure types with different parameters. The simulated data, with different resolution and point densities, represents a good experimental basis to assess the influence of these parameters on the reconstruction of roof models. Besides, roof models with and without outliers can be intensively investigated 
and compared before classifying them from real data using apriori knowledge derived from the PDFs. In this context, the key idea in our paper is the exploitation of latent knowledge encoded in probability density functions in order to distinguish between roof models and the otherwise challenging structures which have been neglected up to now in most approaches.

Our approach consists of a hierarchical pipeline which comprises the detection and the classification of roof structures in 3D point clouds followed by a semantic segmentation using a clustering method and finally the reconstruction of the identified objects. In this context, a variety of methods such as support vector machines (SVM) for the classification, DBSCAN for the density based clustering and $\mathrm{RanSaC}$ for the roof reconstruction are applied. The remainder of this paper is structured as follows: Section 2 gives insights into the most related research. Section 3 elaborates our approach consisting of four main parts: the simulation of roof structures, their classification, the segmentation of roof point clouds and the reconstruction of the identified objects which are presented in sections 3.1, 3.2, 3.3 and 3.4 respectively. Section 4 introduces our conducted experiments and discusses the achieved results. The paper is summarized and concluded in section 5 .

\section{RELATED WORK}

Many researchers dealt with the reconstruction of roofs and their superstructures. Vosselman et al. (2001) combined point clouds and ground plans for the reconstruction of 3D building models based on Hough transform including dormers and chimneys. $\mathrm{Pu}$ and Vosselman (2009) presented a knowledge-based reconstruction method of building models from terrestrial laser scanning data. The general problem, particularly for data-driven approaches, is the challenging reconstruction of dormers, since they are representing a small part of the observations. Kada and Wichmann (2013) presented a feature-driven based approach for the modeling of 3D buildings. Their method detects low-level roof structures, e.g. dormers, using sub-surface segmentation of the input point cloud. Henn et al. (2013) proposed an enhanced version of RanSaC combined with a hierarchical classification using support vector machines leading to a robust roof model estimation even from sparse LiDAR data. The authors of this paper did, however, not explicitly consider roof superstructures such as dormers. In the sense of RanSaC, huge dormers on the roof represent outliers which will be closely investigated in our paper.

Our approach draws upon probability density functions which are used to design suitable features for the classification of roof structures. To this aim, a possible issue to address is to compare the PDFs to each other. In this context, Sakurai et al. (2008) introduced a method which compares two distributions using symmetric and asymmetric Kullback-Leibler divergence as significant value.

The analysis and the interpretation of point clouds has been intensively investigated. Ioannou et al. (2012) introduced an operator based on normal vectors for object recognition in point clouds. They propose the difference of normals (DoN) as a distinct measure for the description of point cloud characteristics depending on a parametrized neighborhood. Jones and Aoun (2009) used histograms based on the angular relationships between a subset of normals for the identification of object classes in a $3 \mathrm{D}$ point cloud.
As mentioned, to study the effect of variable parameters of roofs and their structures, our paper is based on own simulation environment which generates building models with synthetic point clouds. A related paper for the generation of LiDAR point clouds has been presented by Lohani and Mishra (2007).

\section{METHODOLOGY}

This section is presenting an overview on our approach and its related theoretical background. The main components of our method consist of simulating the data, classifying of the roof structures, clustering of the points of these structures and finally their reconstruction.

\subsection{Simulation of roof structures}

In order to examine and analyze the impact of probability densitybased features on the identification and the reconstruction of roofs and their structures, we implemented a building and laser scanning simulation toolbox. On the one hand, the simulation environment consists of a component which allows to generate virtual building models, in particular roofs and roof structures as depicted on the top of Figure 2. On the other hand, the second component consists of a laser scanner simulator which allows for the generation of aerial LiDAR point clouds with different settings, e.g. resolution among others (cf. Figure 2 on the bottom).

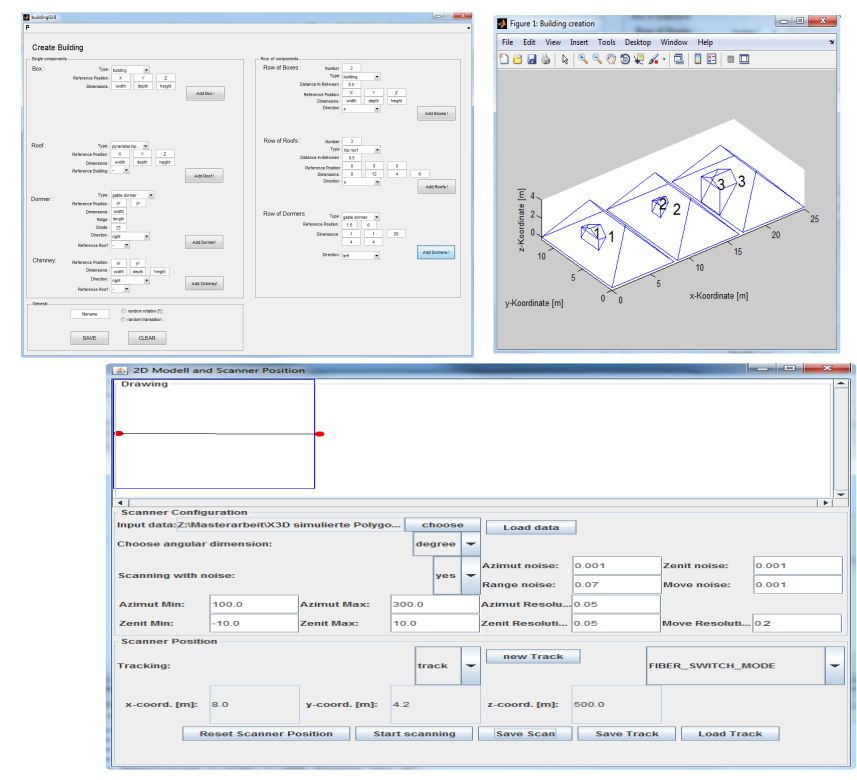

Figure 2. Front-end of the simulation toolbox. Building simulator (top) and laser scanning simulator (bottom).

\subsection{Classification of roof structures}

An important step in our approach is the classification of rooftops and their superstructures, e.g. dormers. To this aim, a skilful choice of discriminant features and predictors is of high importance. The key idea of our method is to design and derive suitable features based on probability density functions. The behaviors of the PDFs are characterizing different roof objects which contributes to their discrimination. To this end, we considered different one-dimensional measures, e.g. roof inclination, derived from the $3 \mathrm{D}$ input point cloud. Based on these measures, each 
PDF is estimated using a non-parametric kernel density estimation (KDE) (Wand and Jones, 1994).

Roof model residuals. For the classification of dormers, the first measure which we consider is the probability density of the residuals of the belonging roof model. This assumes the availability of pre-defined roof models. Accurate roof models allow for an accurate determination of the residuals. Since roof structures represent a white noise in the $3 \mathrm{D}$ point cloud, the $\mathrm{PDF}$ of the residuals is a good tool which reflects their properties. In this context, the residuals are determined using RanSaC (Fischler and Bolles, 1981) as part of the model-driven roof reconstruction method of (Henn et al., 2013). Since the accuracy of the underlying roof model is correlated to the geometry of the belonging superstructure, we investigated their impact on the learning performance including them and in their absence as predictor.

Inclination. Likewise a PDF of the inclination of each point from the point cloud is calculated. To this aim, a surflet (Wahl et al., 2003) is considered, consisting of a pair of a point from the point cloud and its normal vector which represents an approximated plane of its $k$-neighborhood. We choosed $k=5$ in order to take small structures into account. Based on a singular value decomposition (SVD) (Förstner and Wrobel, 2016), the five points with coordinats $\left[x_{i}, y_{i}, z_{i}\right]$ are used to fit a plane whose normal vector is the eigenvector $v_{i}$ associated to the smallest eigenvalue $\sigma_{i}$ of the following matrix $A$ :

$$
\mathbf{A}=\left[\begin{array}{cccc}
x_{1} & y_{1} & z_{1} & 1 \\
\vdots & \vdots & \vdots & \vdots \\
x_{5} & y_{5} & z_{5} & 1
\end{array}\right]=\mathbf{U} \boldsymbol{\Sigma} \mathbf{V}^{T}
$$

The inclination is then calculated based on the $z$-axis of the coordinate system and the surflet for each point.

Mean squared error (MSE). We built also the mean squared error of several measures such as the mentioned point inclination. Figure 3 illustrates a roof point cloud colored according to the MSE values of the point inclinations. The roof on the top includes a shed dormer whereas a gabled dormer is a part of the roof in the bottom.

Angles between normal vector pairs. Following the method of Jones and Aoun (2009), we considered surflet pairs and derived bilateral angles between them. Apart from the angle $\gamma$ between the $z$-axis and a normal vector which is already incorporated in the calculation of the inclination, two further angles $\alpha$ and $\beta$ are considered as illustrated in Figure 4.

Difference of normals (DoN). We make use of the method of Ioannou et al. (2012) which developed an operator dealing with huge unstructured point clouds. This operator calculates the difference of surflets of a given point in two differently sized neighborhoods. This gives the possibility to model the sensitivity regarding to small structures. Figure 5 summarizes the idea of the difference of normals depending on a parametrized radius. In order to acquire a one-dimensional measure, we computed the angle between the resulting difference vector and the $z$-axis.

For the classification task, we designed a set of features in order to discriminate between roof points and dormer points in a first step and between different dormer types in a subsequent step. As stated, we focus in this paper on features based on probability density functions. In this sense, we derive PDFs based on the five mentioned one-dimensional measures for each point from the
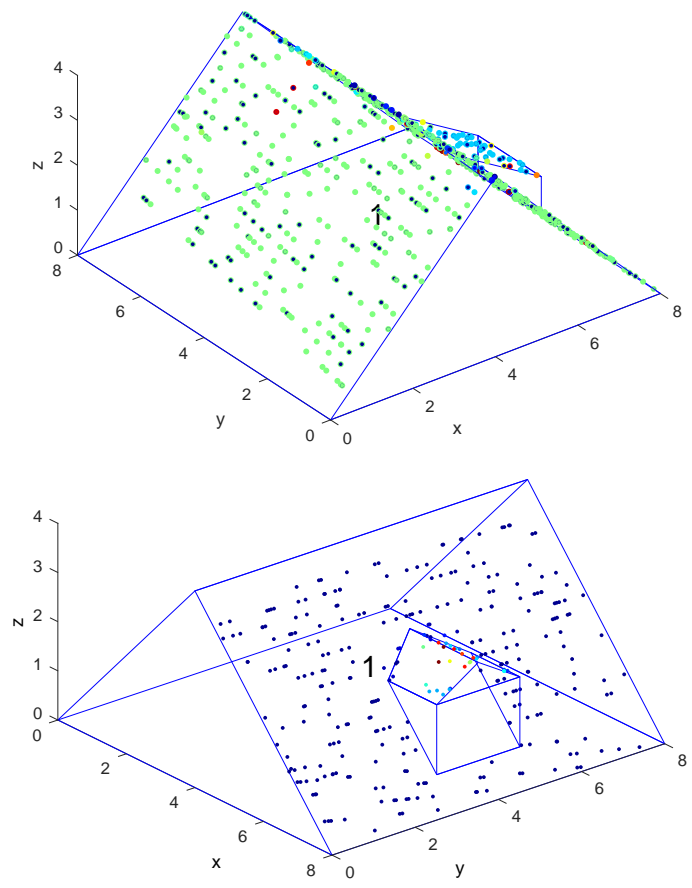

Figure 3. Point cloud colored according to MSE values of the point inclinations. Shed dormer (top) and gabled dormer (bottom).

point cloud. These measures represent the basis for the derivation of the features needed to perform the classification process for the discrimination between the roof structures. In this context, the derived PDF of each measure using KDE is closely investigated considering the following properties:

Skewness. One important property of a probability density function is its skewness which influences its shape and in particular its symmetry. The skewness of the PDF of a data set of $n$ measures $x_{i}$ is defined as follows (Von der Lippe, 2018):

$$
S=\frac{1}{n} \frac{\sum_{i}\left(x_{i}-\bar{x}\right)^{3}}{s^{3}}
$$

where $\bar{x}$ is the mean of $x_{i}$ and $s^{3}$ their standard deviation. According to the value of $S$, we distinguish between left skewed (negative) and right skewed (positive) distributions. A value of zero indicates a symmetric distribution.

The excess kurtosis. A further property of a PDF is the kurtosis which describes its steepness. This measure is calculated based on the fourth moment of the underlying data $x_{i}$ :

$$
K=\frac{1}{n} \frac{\sum_{i}\left(x_{i}-\bar{x}\right)^{4}}{s^{4}}-3 .
$$

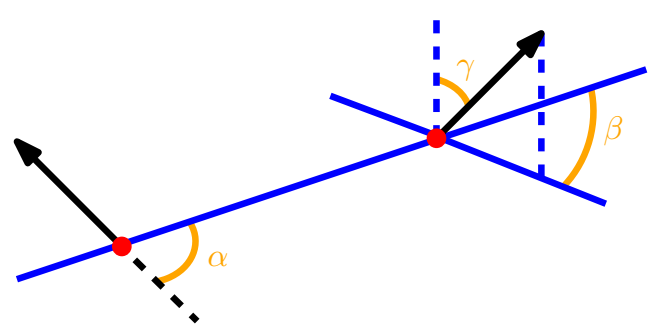

Figure 4. Considered angles between surflet pairs. 


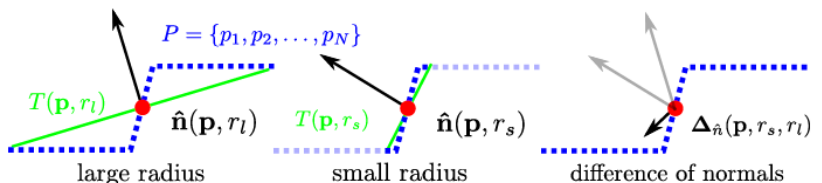

Figure 5. Difference of normals (Ioannou et al., 2012).

The sign of $K$ is an indicator of the steepness. A positive value is characterizing fat-tailed distributions whereas negative values stand for thinner-tailed ones. Symmetric distributions, e.g. normal distribution, have a kurtosis with a zero value.

Kullback-Leibler divergence. We are also interested in comparing two different probability density functions contributing to their discrimination. In this context, the Kullback-Leibler divergence (Kullback and Leibler, 1951) is a suitable measure which quantify how a PDF $p$ is different from another PDF $q$ (Shlens, 2014) based on the information gain as follows:

$$
D_{\mathrm{KL}}(p \| q)=\sum_{i} p_{i} \cdot \log _{2} \frac{p_{i}}{q_{i}}
$$

The formula 4 is specifying the information lost in bits when $q$ is applied to approximate $p$. A symmetric Kullback-Leibler divergence is defined further as the following (Sakurai et al., 2008):

$$
\left.D_{\mathrm{KLS}}(p \| q)\right)=\sum_{i}\left(p_{i}-q_{i}\right) \cdot \log _{2} \frac{p_{i}}{q_{i}} .
$$

Parameters of the PDF. A probability density distribution fitting leads to an approximated parametric PDF of the estimated PDF acquired from the non-parametric kernel density estimation. In this sense, if a normal distribution can be assumed, the parameters $\mu$ and $\sigma$ are exploited and taken as features. Following the spirit of Dehbi and Plümer (2011), an arbitrary distribution which fits the data best can be determined after performing statistical tests, e.g. chi-squared.

Quantile. Further properties of a PDF are reflected by their quantiles dividing the range of the PDF into equally probable continuous intervals. A prominent quantile measure is the well known median. In this context, we partitioned the distribution range in five equally sized parts. We considered the following quintiles (5-quantiles): $0.2,0.4,0.5,0.6$ and 0.8 which is illustrated examplarily for a normal distribution in Figure 6 . These values take part in the feature space for the classification.

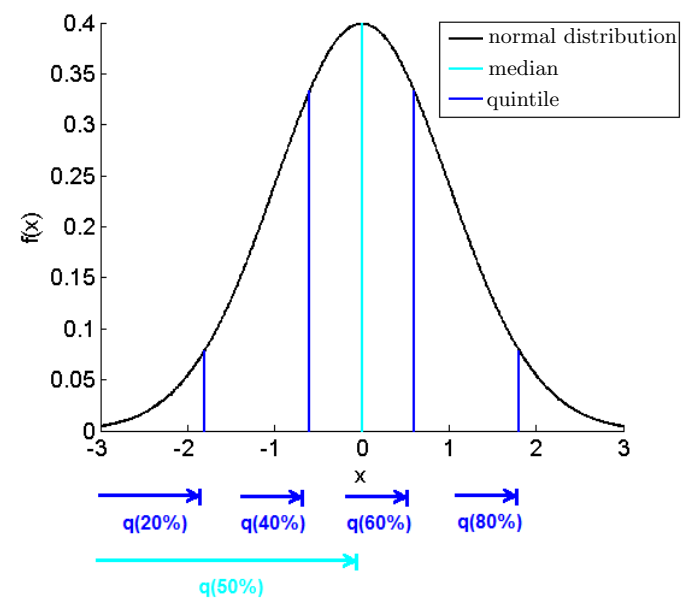

Figure 6. Quintils of a normal distribution.
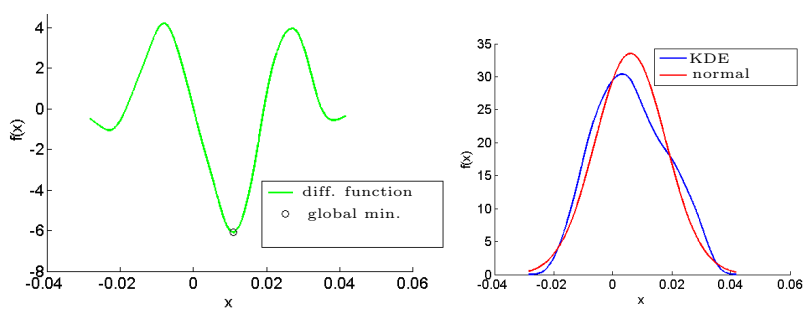

Figure 7. Characteristic deformation of the residual distribution of a roof surface caused by a dormer. An expected normal distribution is taken as reference. Left: Difference function of both distributions with the according global minimum. Right: PDF issued from a KDE (blue) and its approximated normal distribution (red).

Minimum of the difference function. This measure is the global minimum of the difference of two distributions. In this case, a normal distribution is assumed. In this sense, a normal distribution is fitted representing a reference distribution of the PDF estimated by KDE. The difference between these two distributions is then calculated providing its global minimum as additional feature. Figure 7 illustrates a PDF and its approximated normal distribution together with the resulted difference function and the according global minimum.

Based on the pre-designed set of the mentioned features, we followed a hierarchical approach for the classification of the roof dormers as depicted in Figure 8. At first, we differentiate between roofs with and without roof structures in a supervised manner using a binary classification. For this classification, we used support vector machines (SVM) (Vapnik, 1998) as robust classifier. To this aim, we labeled point regions belonging to different roof objects in order to train the classifier. The learning is performed using a 10-fold cross-validation based on simulated data acquired from our implemented simulation toolbox and based on real data from aerial LiDAR point clouds as well. In order to assess the quality of the probability density based features, we performed a feature selection using the Relieff algorithm which calculates a predictor weighting (Robnik-Šikonja and Kononenko, 2003; Kononenko et al., 1997) allowing the selection of the best features with regard to the given class. In this way, redundant features and in particular unsuitable ones are a-priori eliminated. In the second step of our hierarchical classification, only roofs with superstructures are considered. At this stage, we focus on the discrimination between different dormer types, in particular gabled and shed dormers. Since we noticed the existence of roofs with both types, we followed a multi-class classification. We considered three classes: "roof with gabled dormer", "roof with shed dormer" and "roof with gabled and shed dormer". Among others, features related to residuals acquired from an a-priori modelbased roof estimation are used in this supervised learning step.

\subsection{Segmentation of the point cloud}

The last section provides classified roofs including the dormer types. Towards the reconstruction of these acquired objects, we perform a semantic segmentation of the point cloud regarding to the dormer types. To this aim, we conduct a clustering using the density based spatial clustering of applications with noise (DBSCAN) algorithm (Ester et al., 1996). This corresponds to the third step of our hierarchical method (cf. Figure 8). Compared to other clustering methods, DBSCAN does not require the number of clusters to be a-priori known. Further, no assumptions on the cluster shapes have to be made. In this context, we clustered 


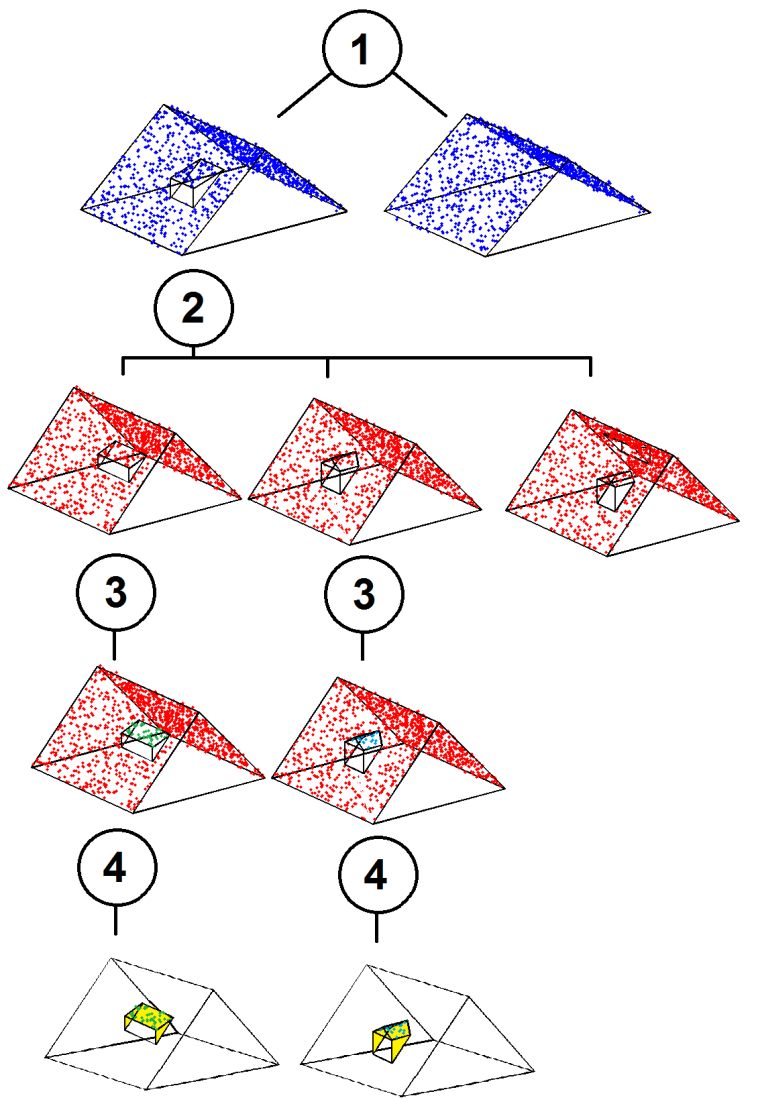

Figure 8. Hierarchical classification and reconstruction of roof structures

again based on one-dimensional measures combined with location information for the reconstruction later on. In the example
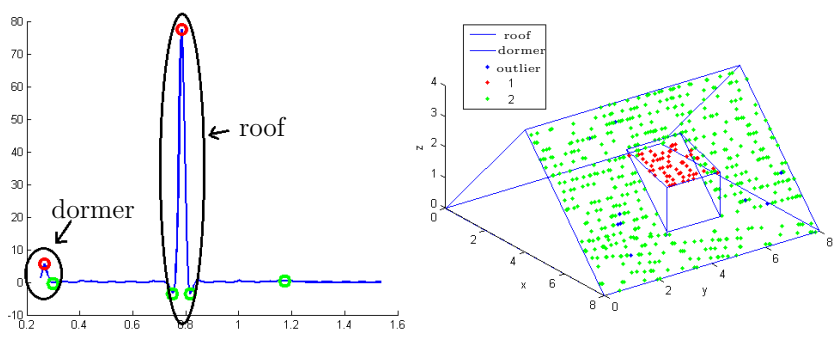

Figure 9. 3D point cloud of a roof colored according to the inclination of the points. Roof points (green) and shed dormer points (red). The PDF of the inclination gives insight into the object type.

of shed dormers, Figure 9 shows a roof point cloud colored according to the point inclinations on the right. The investigation of the PDF of these inclinations reveals that this one-dimensional measure turns out to be, among others, a good prior to distinguish between roof and dormer points. The smaller peak is characterizing dormer points whereas the big one is belonging to roof points. Since we calculated an angle for each point, the clustering results can be associated with the spatial information of the point cloud which is the basis for the reconstruction step.

\subsection{Reconstruction of dormers}

The point clusters acquired from the previous step can be used to reconstruct the roof and dormer surfaces. The roof reconstruction is performed using RanSaC (Fischler and Bolles, 1981) which is expanded in order to consider different roof models based on a catalog of roofs in the spirit of Henn et al. (2013). This derived model can be compared with the roof model estimated from the whole point cloud. In this manner, the influence of white noise on the roof detection and their reconstruction can be investigated. This enables us to compare models stemming from outlier-free point clouds with those derived from point clouds including roof structures. In particular, the simulated point clouds allow for the comparison of these two models providing a true model as ground truth.

The reconstruction of shed dormers is mainly performed using Gauss-Helmert model (Förstner and Wrobel, 2016) estimating a plane $P$ based on the dormer cluster. The boundaries are acquired from a minimal bounding rectangle $m b r$ of the projected points on the $x y$-plane in a first step. This $m b r$ is projected back on the plane $P$ with a ridge parallelity constraint incorporating the $z$-coordinates leading to the final dormer plane $D P$. The rear edge of the shed dormer lies on the intersection of $D P$ and the roof surface. The edge corners are acquired by intersecting the vertical planes with the roof plane. The last parameters of the dormer, i.e. two bottom points, are derived by projecting the front points on the roof plane. Gabled dormers can be reconstructed using RanSaC incorporating a gable roof model and a subsequent projection of the front points on the roof plane.

\section{EXPERIMENTAL RESULTS}

This section describes our experiments comprising the data settings and the achieved results accordingly.

\subsection{Data settings.}

For our experiments, we generated training data consisting of 1700 objects including roofs with and without structures. The inclinations of the dormers vary between $15^{\circ}$ and $45^{\circ}$. Their widths and depths are in the range from $0.5 \mathrm{~m}$ to $4 \mathrm{~m}$ corresponding to detached houses as building type. The point cloud has an average point cloud density between 6 points $/ \mathrm{m}^{2}$ and 11 points $/ \mathrm{m}^{2}$ which corresponds to the resolution of the point cloud from the ISPRS benchmark from Vaihingen (Rottensteiner et al., 2012) and an annotated point cloud from three districts from Dortmund in Germany respectively. In contrast to simulated point clouds, the real data contains noise stemming from facade elements, chimneys or even from other roof parts which have been inaccurately separated. For the annotation of our real data, we make use of orthophotos of the roofs and their belonging footprints for the identification of roof and dormer types. Details to the data and experiments on real data will be subject of a subsequent paper. For our experiments, the LibSVM toolbox has been used (Chang and Lin, 2011) with an RBF kernel.

\subsection{Classification of roofs and dormers}

Simulated data. The classification of synthetic data in roof objects with and without dormers turns out to be very successful. Based on Relieff, the weighted ranking of the features selects those based on the residual (R) distribution and inclination (I) distribution as top predictors. Table 1 shows an excerpt of the best features according to the feature selection. Both residualand inclination-based PDF features turn out to be good for this classification step. These features have been used to train and test the SVM classifier leading to the results summarized in Table 2. 


\begin{tabular}{lc}
\hline Feature & PDF \\
\hline Kurtosis & $\mathrm{R}$ \\
Minimum of difference function & $\mathrm{R}$ \\
Symmetric Kullback-Leibler divergence & $\mathrm{R}$ \\
Asymmetric Kullback-Leibler divergence & $\mathrm{R}$ \\
2nd quintil & $\mathrm{I}$ \\
Median & $\mathrm{I}$ \\
Third quintil & $\mathrm{I}$ \\
1st quintil & $\mathrm{I}$ \\
2nd quintil & $\mathrm{R}$ \\
1st quintil & $\mathrm{R}$ \\
Mean & $\mathrm{I}$ \\
Standard deviation & $\mathrm{R}$ \\
Fourth quintil & $\mathrm{I}$ \\
Skewness & $\mathrm{R}$ \\
.. & $\ldots$ \\
\hline
\end{tabular}

Table 1. Feature selection for simulated data for the classification of roofs with and without structures. I: inclination distribution, $\mathrm{R}$ : residual distribution.

The results of a 10-fold cross-validation as well as the test results on unseen data led to an overall accuracy of more than $98 \%$ for a noise amounting $7 \mathrm{~cm}$. Comparable results have been achieved for a point density of 5.5 points $/ \mathrm{m}^{2}$. For a noise of $14 \mathrm{~cm}$, the class "roof with dormer"' is perfectly classified, poor results have been however achieved for roof without dormers due to high number of false positives. By varying the point density, we noticed that the importance of the inclination related features is decreasing for smaller point densities with more noise which let the classification relying on residual features which needs an a-priori roof model as background knowledge. In order to analyze the impact and the importance of residual information, the residual related features are omitted. Consequently, the classification of roof and dormer objects was unsatisfactory stating that the residual features are indispensable. In our sampled training objects, we took care that we consider dormers and roofs with various parameters. In this context, we varied the width, depth, inclination and the position of dormers on the corresponding roofs. For the choice of the minimal and maximal shape parameter values of dormers and their locations, we followed recommendations satisfying the regulations of the land building directive of the state of North Rhine Westphalia in Germany. Figure 10 shows exemplary the impact of the dormers width on the PDF of the residuals of a gabled roof with the following dimensions $8 \mathrm{~m} \times 12 \mathrm{~m} \times 4 \mathrm{~m}$. Herewith, the ridge is directed along the longest side of the roof. Not only the variation of the dormer widths but also the variation of other parameters have shown that the width of the residual's PDF is higher for bigger dormers. The bend in the PDF of the roof without dormer is characterizing the ridge of the roof.

\begin{tabular}{|c||l||l|l||c|}
\hline \multicolumn{2}{|c||}{} & \multicolumn{2}{c|}{ reference } & precision \\
with & $\begin{array}{l}\text { without } \\
\text { dormer }\end{array}$ & \\
dormer & \\
\hline \hline results & $\begin{array}{l}\text { with } \\
\text { dormer }\end{array}$ & 999 & 1 & $99.9 \%$ \\
\cline { 2 - 5 } & $\begin{array}{l}\text { without } \\
\text { dormer }\end{array}$ & 0 & 400 & $100 \%$ \\
\hline \hline recall & & $100 \%$ & $99.8 \%$ & $98.9 \%$ \\
\hline
\end{tabular}

Table 2. Results of the binary classification of roofs and dormers based on simulated data. Point density: 11 points $/ \mathrm{m}^{2}$, noise: $7 \mathrm{~cm}$.

\begin{tabular}{lc}
\hline Feature & PDF \\
\hline Median & $\mathrm{I}$ \\
2nd quintil & $\mathrm{I}$ \\
3rd quintil & $\mathrm{I}$ \\
Mean & $\mathrm{I}$ \\
Symmetric Kullback-Leibler divergence & $\mathrm{R}$ \\
1st quintil & $\mathrm{I}$ \\
Excess & $\mathrm{R}$ \\
Minimum of difference function & $\mathrm{R}$ \\
4th quintil & $\mathrm{R}$ \\
\hline
\end{tabular}

Table 3. Feature selection for the multi-class classification of dormer types (gabled, shed and both) based on simulated data.

I: inclination distribution, R: residual distribution.

Real data. We applied the trained classifier from the simulated point cloud on 58 buildings from the mentioned real data from Dortmund. This leads, however, to poor results. For this reason, we trained a new classifier on the real data using the same features and applying a new feature selection. In this manner, we got very good and promising results which be subject of an own subsequent paper with deeper empirical discussions and explanations.

\subsection{Classification of dormer types}

Since dormer types are hardly distinguishable in orthophotos due to shadows and insufficient resolution we used only simulated data for the classification of the dormer types. We performed a multi-class classification to distinguish between roofs including shed dormers or gabled dormers or both of them. The feature selection revealed that residual and inclination based features are also important for this task as depicted in Table 3. Particularly, we stated that the inclination is discriminative for shed dormers rather than gabled dormers. Omitting the residual distributions leads to the same effect on the results as it was the case in the previous classification task. The classification results are shown in tables 4 and 5 for different point densities, namely 11 and 7.5 points $/ m^{2}$ respectively.

\begin{tabular}{|c||c||c|c|c||c|}
\hline \multicolumn{2}{|c||}{} & \multicolumn{3}{c|}{ reference } & precision \\
\multicolumn{2}{|c|}{} & gabled & deshed & both & \\
\hline \hline \multirow{3}{*}{ results } & gabled & 403 & 20 & 15 & $92.0 \%$ \\
\cline { 2 - 5 } & deshed & 13 & 401 & 10 & $94.6 \%$ \\
\cline { 2 - 5 } & both & 0 & 1 & 242 & $99.6 \%$ \\
\hline \hline recall & & $96.9 \%$ & $95.0 \%$ & $90.6 \%$ & $94.7 \%$ \\
\hline
\end{tabular}

Table 4. multi-class classification results of dormer types using a 10 -fold cross validation. Point density: 11 points $/ \mathrm{m}^{2}$.

We performed also a binary classification for the discrimination between the two dormer types. For this task, the kurtosis of the residuals and the $\alpha$-values turn out to be the most important features. Features issued from the distribution of DoN, $\alpha$ - and $\beta$ -

\begin{tabular}{|c||c||c|c|c||c|}
\hline \multicolumn{2}{|c||}{} & \multicolumn{3}{c||}{ reference } & precision \\
\multicolumn{2}{|c||}{ gabled } & deshed & both & \\
\hline \hline \multirow{3}{*}{ results } & gabled & 397 & 41 & 34 & $84.1 \%$ \\
\cline { 2 - 5 } & deshed & 28 & 382 & 12 & $90.5 \%$ \\
\cline { 2 - 5 } & both & 4 & 0 & 211 & $98.1 \%$ \\
\hline \hline recall & & $92.5 \%$ & $90.3 \%$ & 82.1 & $89.3 \%$ \\
\hline
\end{tabular}

Table 5. multi-class classification results of dormer types using a 10-fold cross validation. Point density: 7.5 points $/ \mathrm{m}^{2}$. 

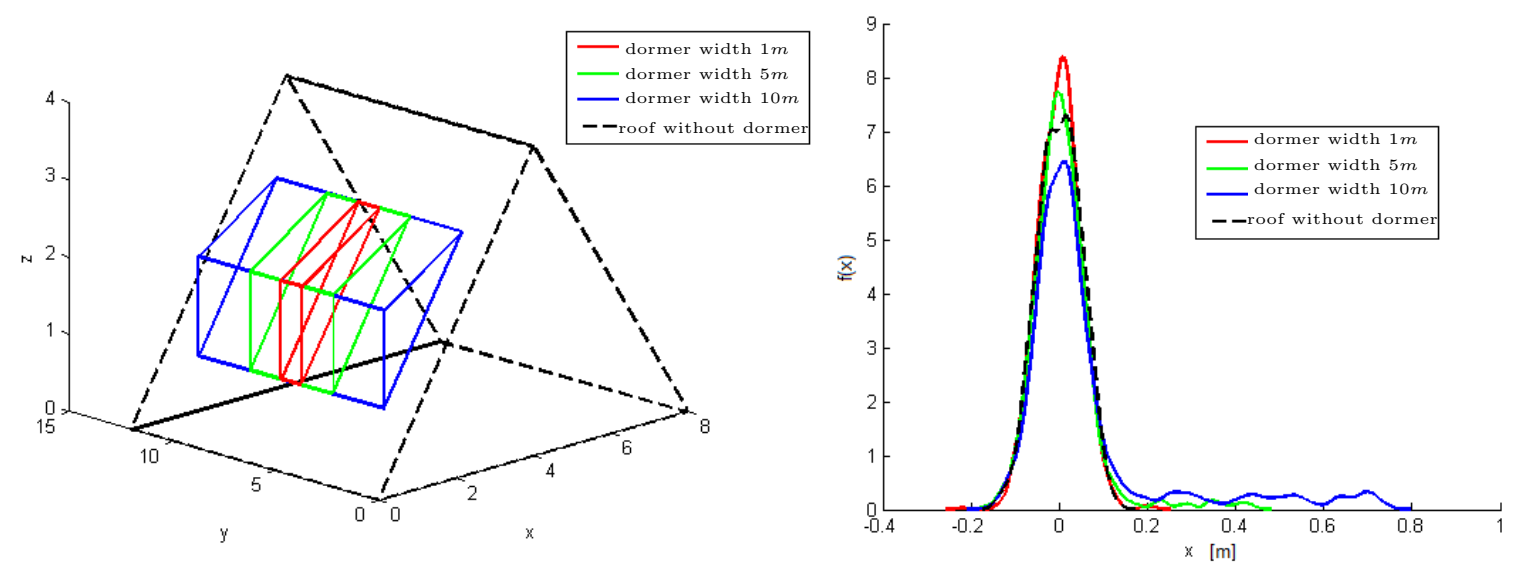

Figure 10. Impact of varying dormer widths (left) on the PDF of residuals (right).

\begin{tabular}{lc}
\hline Feature & PDF \\
\hline Kurtosis & $\mathrm{R}$ \\
Kurtosis & $\alpha$ \\
Standard deviation & $\mathrm{R}$ \\
Minimum of difference function & $\mathrm{R}$ \\
Mean & $\mathrm{I}$ \\
Skewness & $\mathrm{I}$ \\
1st quintil & $\mathrm{I}$ \\
Asymmetric Kullback-Leibler divergence & DoN \\
Symmetric Kullback-Leibler divergence & $\beta$ \\
Mean & $\mathrm{R}$ \\
Symmetric Kullback-Leibler divergence & DoN \\
2nd quintil & $\alpha$ \\
Symmetric Kullback-Leibler divergence & $\mathrm{I}$ \\
\hline
\end{tabular}

Table 6. Feature selection for the binary classification of dormer types (gabled and shed) based on simulated data. I: inclination distribution, R: residual distribution.

angles turn out to be also required. Table 6 shows the most relevant features for this classification task. In a further step, we incorporated chimneys in the simulated data for the assessment of their influence on the classification. The classification results revealed that these structures did not have a significant impact on the classifier performance.

\subsection{Clustering and reconstruction of roof structures}

The use of residuals as measures in DBSCAN leads to the most successful results. That means, however, that model-knowledge of the roof is required. Other one dimensional measures have been used to segment the point cloud without requiring the knowledge of the roof model. The inclinations and their MSEs are in this sense empirically the most appropriate ones. They are, however, sensitive to noise and influenced by points near the roof ridge as illustrated by Figure 11. In order to deal with this issue, a second DBSCAN clustering has been performed based on the $3 \mathrm{D}$ coordinates of the points leading to good results for not too sparse point sets. Especially, dormer positions are relevant at this stage. Dormers near to ridge are falsely associated to ridge regions for a low point cloud density. All in all, for a density of 11 points $/ \mathrm{m}^{2}$ good clustering results are achieved enabling a reconstruction of dormers subsequently. The reconstruction of the dormers depends on the clustering step which in turn depends on the dormer positions and the point density. This influences the determination of the minimum bounding rectangles enclosing the dormer points. Outliers lead in this context to oversized dormers.

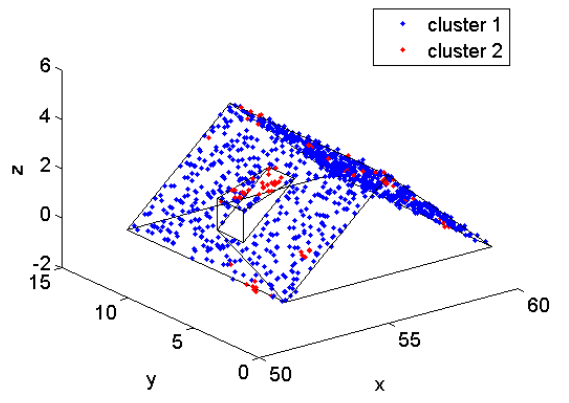

Figure 11. Point cloud clustering based on inclination and mean square error (MSE). Ridge points share the same cluster with dormer points.

Figure 12 illustrates the reconstruction results of shed dormers for different point densities. Red points have been acquired after performing the second DBSCAN clustering based on spatial information of the points.

In order to assess the impact of dormers and roof superstructures in general on the quality of roof models estimated by RanSaC, we compared the deviation of inclinations between such roof models and reference models. The results are summarized in Table 7. It can be stated that roof models without dormers are identified more accurately than those including dormers which confirm their influence on the estimation. A higher noise does not have a negative impact on the resulted models which explains the robustness of $\mathrm{RanSaC}$ against noise.

\begin{tabular}{c|cc|cc|c}
\hline $\begin{array}{c}\text { point density } \\
{\left[\text { Punkte } / \mathrm{m}^{2}\right]}\end{array}$ & \multicolumn{2}{|c|}{ roof with dormer } & \multicolumn{2}{|c|}{ roof without dormer } & range noise \\
\hline 11 & -0.04 & 0.30 & -0.14 & 0.23 & 14 \\
11 & -0.40 & 0.54 & -0.45 & 0.40 & 7 \\
7.7 & -0.39 & 0.51 & -0.45 & 0.37 & 7 \\
6.6 & -0.40 & 0.50 & -0.42 & 0.39 & 7 \\
5.5 & -0.41 & 0.51 & -0.41 & 0.40 & 7 \\
4.4 & -0.40 & 0.54 & -0.46 & 0.38 & 7 \\
3.3 & -0.39 & 0.51 & -0.45 & 0.40 & 7 \\
\hline
\end{tabular}

Table 7. Deviations between the estimated roof models using $\mathrm{RanSaC}$ and the true models. Mean and standard deviation of the differences with regard to the inclinations depending on the point density and noise are calculated. 

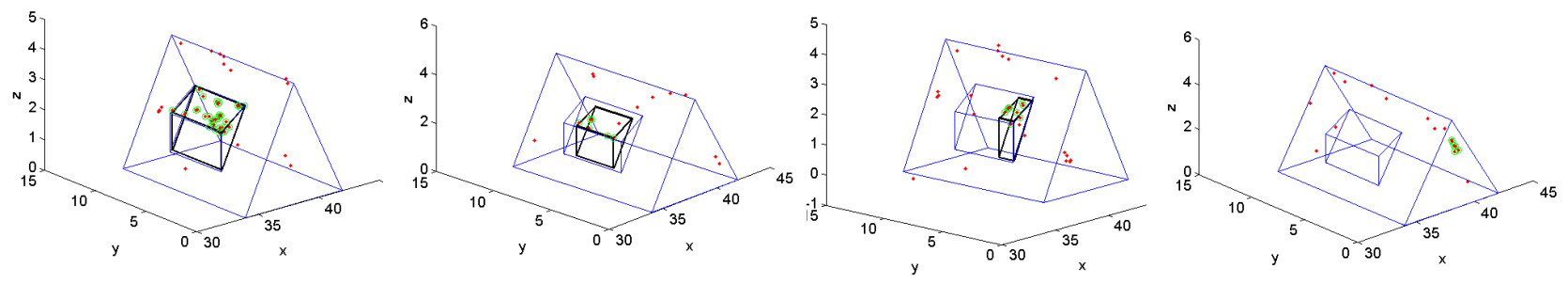

Figure 12. Reconstruction of a shed dormer with different point densities: from left to right 11, 5.5, 4.4 and 3.3 points $/ \mathrm{m}^{2}$. Reconstructed dormer (black). MSE and inclination based clustering results (red). Clustered region after new clustering of the red points based on their 3D coordinates (green).

\section{CONCLUSION AND OUTLOOK}

This paper introduced an approach for the automatic classification and reconstruction of roofs and their structures. A hierarchical classification method is followed which discriminates between roofs and dormers followed by a classification of different dormer types subsequently. Both classification steps leads to very good results up to $99 \%$. The key idea is the use of skilful features issued from probability density functions (PDFs) of specific measures from the point cloud. PDFs of inclinations and the residuals of model-based generated roofs via RanSac are used among others. Even without residual information, a clustering using DBSCAN leads to the identification and reconstruction of dormers.

The classification and reconstruction results are presented based on simulated and real data. The paper also introduces an implemented simulation toolbox which gives the possibility to generate different roof and dormer models with various and controlled parameters. For future work, additional roof and dormer types can be considered. Correspondingly, the investigation of additional features is envisaged.

\section{ACKNOWLEDGEMENTS}

The authors thank Dirk Dörschlag for the implementation of the laser scanning simulator.

\section{REFERENCES}

Biljecki, F., Stoter, J., Ledoux, H., Zlatanova, S. and ltekin, A., 2015. Applications of 3d city models: State of the art review. ISPRS Inter-national Journal of Geo-Information 4(4), pp. 2842-2889.

Chang, C.-C. and Lin, C.-J., 2011. Libsvm: A library for support vector machines. ACM Trans. Intell. Syst. Technol. 2(3), pp. 27:1-27:27.

Dehbi, Y. and Plümer, L., 2011. Learning grammar rules of building parts from precise models and noisy observations. ISPRS Journal of Photogrammetry and Remote Sensing 66(2), pp. 166-6176. Quality, Scale and Analysis Aspects of Urban City Models.

Ester, M., Kriegel, H.-P., Sander, J. and Xu, X., 1996. A Density-Based Algorithm for Discovering Clusters in Large Spatial Databases with Noise. In: Knowledge Discovery and Data Mining, pp. 226-231.

Fischler, M. A. and Bolles, R. C., 1981. Random sample consensus: A paradigm for model fitting with applications to image analysis and automated cartography. Communications of the ACM 24 (6), pp. 381395 .

Förstner, W. and Wrobel, B., 2016. Photogrammetric Computer Vision: Geometry, Orientation and Reconstruction. Geometry and Computing, Springer International Publishing.

Gröger, G., Kolbe, T. H., Nagel, C. and Häfele, K.-H., 2012. OpenGIS City Geography Markup Language (CityGML) Encoding Standard. Version 2.0.0, Open Geospatial Consortium, OGC Doc. No. 12-019.

Henn, A., Gröger, G., Stroh, V. and Plümer, L., 2013. Model driven reconstruction of roofs from sparse LIDAR point clouds. ISPRS Journal of Photogrammetry and Remote Sensing 76, pp. 17-29.
Ioannou, Y., Taati, B., Harrap, R. and Greenspan, M., 2012. Difference of normals as a multi-scale operator in unorganized point clouds. In: 2012 Second International Conference on 3D Imaging, Modeling, Processing, Visualization \& Transmission, IEEE, pp. 501-508.

Jones, B. and Aoun, M., 2009. Learning 3d point cloud histograms. CS229 Machine Learning Project.

Kada, M. and Wichmann, A., 2013. Feature-driven 3d building modeling using planar halfspaces. ISPRS Annals of Photogrammetry, Remote Sensing and Spatial Information Sciences II-3/W3, pp. 37-42.

Kononenko, I., Šimec, E. and Robnik-Šikonja, M., 1997. Overcoming the myopia of inductive learning algorithms with relieff. Applied Intelligence 7(1), pp. 39-55.

Kullback, S. and Leibler, R. A., 1951. On information and sufficiency. The annals of mathematical statistics 22(1), pp. 79-86.

Lohani, B. and Mishra, R. K., 2007. Generating LiDAR Data in laboratory: LiDAR simulator. In: ISPRS Workshop on Laser Scanning 2007 and SilviLaser 2007, Finnland.

Musialski, P., Wonka, P., Aliaga, D. G., Wimmer, M., van Gool, L. and Purgathofer, W., 2012. A Survey of Urban Reconstruction. In: EUROGRAPHICS 2012 State of the Art Reports, Eurographics Association, pp. 1-28.

$\mathrm{Pu}$, S. and Vosselman, G., 2009. Knowledge based reconstruction of building models from terrestrial laser scanning data. ISPRS Journal of Photogrammetry and Remote Sensing 64(6), pp. 575-584.

Robnik-Šikonja, M. and Kononenko, I., 2003. Theoretical and empirical analysis of relieff and rrelieff. Mach. Learn. 53(1-2), pp. 23-69.

Rottensteiner, F., Sohn, G., Jung, J., Gerke, M., Baillard, C., Benitez, S. and Breitkopf, U., 2012. The isprs benchmark on urban object classification and $3 \mathrm{~d}$ building reconstruction. ISPRS Ann. Photogramm. Remote Sens. Spat. Inf. Sci 1(3), pp. 293-298.

Sakurai, Y., Li, L., Chong, R. and Faloutsos, C., 2008. Efficient distribution mining and classification. In: Proceedings of the 2008 SIAM international conference on data mining, SIAM, pp. 632-643.

Shlens, J., 2014. Notes on kullback-leibler divergence and likelihood. arXiv preprint arXiv:1404.2000.

Vapnik, V. N., 1998. Statistical learning theory. A Wiley-Interscience publication, Wiley, New York, USA.

Von der Lippe, P., 2018. Deskriptive Statistik. Walter de Gruyter GmbH \& Co KG.

Vosselman, G., Dijkman, S. et al., 2001. 3d building model reconstruction from point clouds and ground plans. International archives of photogrammetry remote sensing and spatial information sciences 34(3/W4), pp. 37-44

Wahl, E., Hillenbrand, U. and Hirzinger, G., 2003. Surflet-pair-relation histograms: a statistical 3d-shape representation for rapid classification. In: Proceedings of Fourth International Conference on 3-D Digital Imaging and Modeling, 2003. 3DIM 2003, pp. 474-481.

Wand, M. P. and Jones, M. C., 1994. Kernel smoothing. Crc Press.

Wang, R., Peethambaran, J. and Chen, D., 2018. Lidar point clouds to 3-d urban models : a review. IEEE Journal of Selected Topics in Applied Earth Observations and Remote Sensing 11(2), pp. 606-627. 